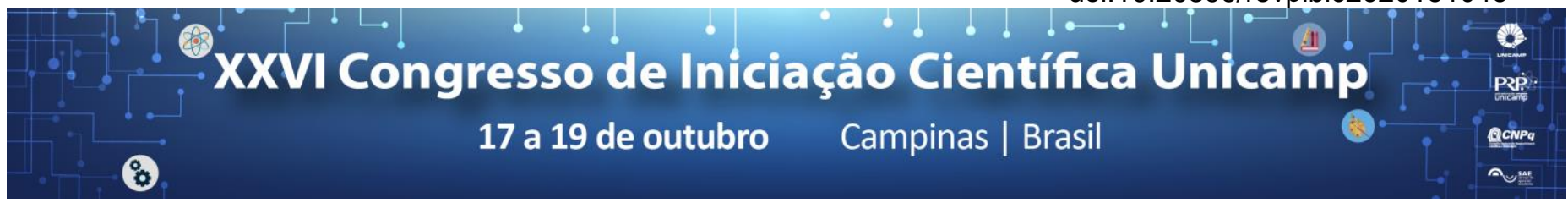

\title{
Modeling and simulation of microreactor with static and perforated elements for biodiesel synthesis
}

\author{
Matheus S. C. Celestino*, João L. Silva Jr., Osvaldir P. Taranto, Harrson S. Santana.
}

\begin{abstract}
This study compares the performance between micromixers with static elements $(\mathrm{MO})$ and micromixers with perforated elements (MPO) in order to develop microscale mixing mechanisms and to improve the performance of chemical reactions, such as the biodiesel production. A computational study was carried out to analyze the performance of these new micromixers with the mixing of the reagents sunflower oil and ethanol, and also the synthesis of biodiesel from these fluids. The results were expressed in terms of the mixing index (degree of mixing of the reactants) and their conversion into biodiesel. High mixing indexes ( 0.99) were obtained, mainly for MO. In relation to the chemical reaction, the highest value reached was $97 \%$ conversion in a residence time of $180 \mathrm{~s}$.
\end{abstract}

\section{Key words:}

Micromixer, Microreactor, Microfuidics.

\section{Introduction}

The microfluidics presents itself as a promising area, due to its advantages when compared to traditional macroscopic processes ${ }^{1}$. Accordingly, studies about it have been important for the process of system miniaturization, organic synthesis and energy production, aiming to the creation of technologies that may be more advantageous than those of the present. Among the advantages of these microfluidic systems are the smaller amount of reagents and samples, reduced residence times, low manufacturing costs, high surface area-tovolume ratio and high rates of heat and mass transfer. Processes that occur in microdevices are usually in laminar regime (that is, the flow is marked by the molecular motion in layers), which in some cases may hinder the mixing of components and, consequently result in poor reactive efficiency. In order to overcome this issue, researchers have been developing in recent years several microdevices which use the most varied types of micromixers to intensify the species interaction and the mass transfer rates.

Hence, the development of processes in this area can lead to an important improvement in the optimization of production processes. Thereby, the present research evaluated the optimization of micromixers with static obstacles (MO) and with perforated obstacles (MPO) to verify the best interaction and mass transfer between them for further application in the biodiesel synthesis from the transesterification reaction of the sunflower vegetable oil with ethanol / $\mathrm{KOH}$.

\section{Results and Discussion}

Numerical simulations were performed to evaluate the mixing degree between the reactants (i.e. a mixing index calculated from the standard deviation of the vegetable oil mass fraction) for a Reynolds number range of $0.1-100$, and the chemical reaction yield (evaluated for the vegetable oil conversion, i.e., relationship between oil molar concentration at inlet and outlet of the microdevices), for a residence time range from 10 to 180 $\mathrm{s}$. Three different heights of the micromixers $(0.2,1$ and 2 $\mathrm{mm}$ ) were investigated. The new micromixers and the results are shown in Figure 1.

The MO design exhibited superior performance, providing a maximum mixing index of 0.99 and an oil conversion of $97 \%$, both for the height of $0.2 \mathrm{~mm}(200$ $\mu \mathrm{m})$.
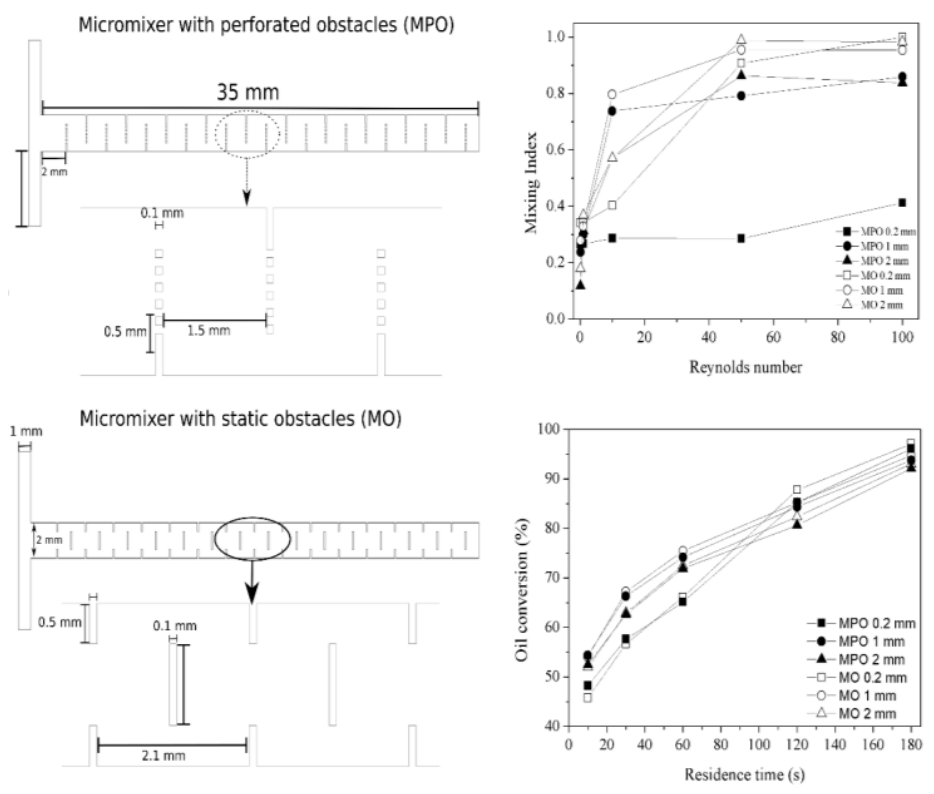

Figure 1. Design and dimensions $(\mathrm{mm})$ of micromixers (left) and numerical results (right)

\section{Conclusions}

The MO achieved higher blends with $97 \%$ sunflower oil conversion, presenting superior performance for biodiesel production from sunflower oil with ethanol.

\section{Acknowledgement}

We are grateful to PIBIC and FAPESP (Process 2016/20842-4) for its support in carrying out this work.

${ }^{1}$ SANTANA, H. S.; TORTOLA, S.; REIS, É. M.; SILVA, J. L.; TARANTO, O. P. Transesterification reaction of sunflower oil and ethanol for biodiesel synthesis in microchannel reactor: Experimental and simulation studies. Chemical Engineering Journal, v. 302, p. 752-762, 2016 